\title{
openheart Childhood and adult exposure to secondhand tobacco smoke and cardiac structure and function: results from Echo-SOL
}

\author{
Melissa Suzanne Burroughs Peña, ${ }^{1}$ Katrina Swett, ${ }^{2}$ Robert C Kaplan, ${ }^{3}$ \\ Krista Perreira, ${ }^{4}$ Martha Daviglus, ${ }^{5}$ Mayank M Kansal, ${ }^{6}$ Jianwen Cai, ${ }^{7}$ \\ Aida L Giachello, ${ }^{8}$ Marc D Gellman, ${ }^{9}$ Eric J Velazquez, ${ }^{10}$ Carlos J Rodriguez ${ }^{2}$
}

\begin{abstract}
- Additional material is published online only. To view please visit the journal online (http://dx.doi.org/10.1136/ openhrt-2018-000831).
\end{abstract}

To cite: Burroughs Peña MS, Swett K, Kaplan RC, et al. Childhood and adult exposure to secondhand tobacco smoke and cardiac structure and function: results from Echo-SOL. Open Heart 2018;5:e000831. doi:10.1136/ openhrt-2018-000831

Received 6 April 2018 Revised 22 July 2018 Accepted 20 September 2018

Check for updates

(C) Author(s) (or their employer(s)) 2018. Re-use permitted under CC BY-NC. No commercial re-use. See rights and permissions. Published by BMJ.

For numbered affiliations see end of article.

Correspondence to Dr Melissa Suzanne Burroughs Peña; mburroughspena@ stanfordhealthcare.org

\section{ABSTRACT}

Objective To describe the relationship of household secondhand smoke (SHS) exposure and cardiac structure and function.

Methods Participants ( $n=1069 ; 68 \%$ female; age 45-74 years) without history of tobacco use, coronary artery disease or severe valvular disease were included. Past childhood (starting at age $<13$ years), adolescent/adult and current exposure to household SHS was assessed. Survey linear regression analyses were used to model the relationship of SHS exposure and echocardiographic measures of cardiac structure and function, adjusting for covariates (age, sex, study site, alcohol use, physical activity and education).

Results SHS exposure in childhood only was associated with reduced $E / A$ velocity ratio ( $\beta=-0.06$ (SE 0.02 ), $\mathrm{p}=0.008$ ). SHS exposure in adolescence/adult only was associated with increased left ventricular ejection fraction (LVEF) (1.2 (0.6), $p=0.04)$, left atrial volume index (1.7 (0.8), $p=0.04$ ) and decreased isovolumic relaxation time $(-0.003(0.002), p=0.03)$. SHS exposure in childhood and adolescence/adult was associated with worse left ventricular global longitudinal strain (LVGLS) (two-chamber) (0.8 (0.4), $p=0.049)$. Compared with individuals who do not live with a tobacco smoker, individuals who currently live with at least one tobacco smoker had reduced LVEF ( $-1.4(0.6), p=0.02)$, LVGLS (average) $(0.9(0.40), p=0.03)$, medial $E^{\prime}$ velocity $(-0.5$ $(0.2), p=0.01)$, E/A ratio $(-0.09(0.03), p=0.003)$ and right ventricular fractional area change $(-0.02(0.01), p=0.01)$ with increased isovolumic relaxation time $(0.006(0.003)$, $\mathrm{p}=0.04$ )

Conclusions Past and current household exposure to SHS was associated with abnormalities in cardiac systolic and diastolic function. Reducing household SHS exposure may be an opportunity for cardiac dysfunction prevention to reduce the risk of future clinical heart failure.

\section{INTRODUCTION}

Worldwide, secondhand smoke (SHS) from tobacco accounts for 603000 deaths, representing $1 \%$ of total worldwide mortality, with ischaemic heart disease representing the

\section{Key questions}

What is already known about this subject?

- Household secondhand tobacco smoke exposure has implications for cardiopulmonary health across generations in a household. The association of secondhand tobacco smoke exposure with adverse changes in cardiac structure and function has not been described.

What does this study add?

- In this observational study of a population-based cohort of US Hispanics/Latinos, self-reported chronic exposure to household secondhand smoke in childhood and adolescence/adulthood was associated with worse left ventricular longitudinal strain. Individuals who reported living with one smoker who smokes in the home had worse left ventricular diastolic and systolic function.

How might this impact on clinical practice?

- Reducing household secondhand tobacco smoke exposure is an opportunity for prevention of cardiac dysfunction to reduce risk of possible future clinical heart failure.

largest percentage of mortality. ${ }^{1}$ While men are more likely to smoke tobacco, female non-smokers and children are more likely to be exposed to SHS compared with male non-smokers. ${ }^{1}$ Epidemiological data on the health hazards of SHS exposure resulted in the enactment of public policy that restricted the use of tobacco products in public places including restaurants, bars and hotels. ${ }^{2-7}$ Reducing exposure to SHS in public places has reduced the incidence of acute coronary syndromes throughout USA. ${ }^{8-12}$ However, while much attention has been directed to reducing acute exposure to tobacco smoke in public places, less attention has been paid to chronic exposure to tobacco smoke in the household. 
Hispanics/Latinos in the USA are a heterogeneous population with patterns of tobacco use that vary by national background. While Mexican-Americans tend to have lower prevalence of tobacco use compared with non-Hispanic whites, this trend is not uniform among all Hispanics/Latinos, such as Cubans and Puerto Ricans who report higher tobacco use. $^{13-15}$ Overall, 26\% of men and $15 \%$ of women in the Hispanic Community Health Study/Study of Latinos (HCHS/SOL) were current tobacco smokers, while 32\% of Puerto Rican women and $21 \%$ of Cuban women reported ongoing tobacco use. Additionally, household SHS was reported by $40 \% \mathrm{HCHS} / \mathrm{SOL}$ participants, a finding that has implications for cardiopulmonary health across generations in a household. ${ }^{14}{ }^{16}$ While SHS exposure has been steadily declining in the USA, the decline in SHS exposure among Hispanics/Latinos lags behind the observed decline in non-Hispanic whites. ${ }^{17}$ The potential impact of chronic household SHS exposure at various points in the life course on cardiac structure and function has not been well described but has particular relevance for Hispanic/Latino populations who present with heart failure at an earlier age than non-Hispanic whites. ${ }^{18}$ The objective of this study was to determine the association of past childhood/adult and current SHS exposure with cardiac structure and function as measured by echocardiography among Hispanics/Latinos.

\section{METHODS \\ Study setting}

HCHS/SOL is a population-based study of self-identified Hispanic/Latino men and women ( $\mathrm{N}=16$ 415) aged 18-74 years. ${ }^{19}{ }^{20} \mathrm{HCHS} / \mathrm{SOL}$ was designed to assess chronic disease in Hispanic/Latino individuals living in four cities in the USA: Bronx, New York; Chicago, Illinois; Miami, Florida; and San Diego, California. The details of HCHS/SOL sampling and recruitment methods have been previously described. ${ }^{19}$ Exclusion criteria included: active-duty military service, not living at the residential address, planning to move from the area within 6 months, unable to complete the study in English or Spanish or physically unable to attend the clinic examination.

Echo-SOL is an ancillary study of HCHS/SOL consisting of 1824 participants recruited through a stratified-sampling process representative of the parent study. ${ }^{21}$ Echo-SOL was designed to characterise cardiac structure and function using echocardiography in a representative sample of Hispanic/Latino individuals living in the USA. The baseline Echo-SOL echocardiography examination was performed from 2011 to $2014 .^{21}$ Eligibility criteria for Echo-SOL included: age 45 years or older; self-reported Hispanic/Latino of Mexican, Puerto Rican, Cuban, Dominican, Central American or South American background; and enrolment 36 months or fewer from the date of the baseline HCHS/SOL visit. Echo-SOL enrolled on average $\sim 80 \%$ of eligible participants. The Institutional Review Board at each study site provided approval and oversight of all study materials and activities. All Echo-SOL participants gave informed consent.

Phillips IE-33 or Sonos 5500/7500 ultrasound imaging platforms were used in the acquisition of all echocardiographic imaging data. A standard transthoracic echocardiography examination was performed with the participant in the partial left decubitus position. Two-dimensional (2D), spectral, colour flow and tissue Doppler images were acquired in the parasternal long axis, short axis and apical four-chamber and two-chamber long-axis views. Left ventricular (LV) global longitudinal strain (GLS) analysis was performed using the vendor-independent Cardiac Performance Analysis software (TomTec, Hamden, Connecticute, USA) on acquired 2D images. All images were read by a Registered Diagnostic Cardiac Sonographer and over-read by a cardiologist (CJR) with level 3 advanced echocardiography training. Inter-reader and intrareader variability were assessed and determined to have a high degree of interclass correlation for each measurement (0.80-0.99).

\section{Definitions}

Chronic SHS exposure was assessed by questionnaire, which was available in both English and Spanish. SHS was characterised in the following manner:

1. Any chronic exposure to household SHS: any chronic exposure to household SHS was defined as answering yes to any of the following items: before age 13 years, have you ever lived with a regular smoker who smoked in the home?, or since age 13 years, have you ever lived with a regular smoker who smoked in the home?

2. Only chronic childhood exposure to household SHS: chronic childhood exposure to household SHS was defined as answering yes to following question: before age 13 years, have you ever lived with a regular smoker who smoked in the home?; excluding participants who answered yes to the question: since age 13 , have you ever lived with a regular smoker who smoked in the home?

3. Only chronic adolescent/adult exposure to household SHS: chronic adolescent/adult exposure to household SHS was defined as answering yes to the following question: since age 13 years, have you ever lived with a regular smoker who smoked in the home?; excluding participants who answered yes to the question: before age 13 years, have you ever lived with a regular smoker who smoked in the home?

4. Chronic childhood and adolescent/adult exposure to household SHS: chronic childhood and adolescent/adult exposure to household SHS was defined by answering yes to both of the following two questions: (1) before age 13 years, have you ever lived with a regular smoker who smoked in the home, and (2) since age 13 years, have you ever lived with a regular smoker who smoked in the home?

5. Current SHS exposure

a. Participants were asked to specify the number of individuals in the household who currently regularly 
smoke tobacco in the home. This variable was parameterised into three categories: 0,1 and 2+.

b. Participants were asked to specify the number of hours per week that they were in close contact with individuals actively smoking tobacco in any location (ie, house, work and car). This variable was parameterised into three categories: 0,1 to the median and above the median.

The echocardiographic measurements included in this study included multiple measures of left and right heart structure and function: LV mass indexed to body surface area, LV end-diastolic volume, LV end-systolic volume, LV ejection fraction (LVEF), LV stroke volume, medial and lateral tissue Doppler E' velocities, mitral inflow E/A ratio, $\mathrm{E} / \mathrm{E}^{\prime}$ ratio, isovolumic relaxation time (IVRT), peak right ventricular/right atrial pressure gradient, tricuspid annular plane systolic excursion, right ventricular fractional area change, left atrial volume index, LV GLS (four-chamber view, two-chamber view and average).

Echo-SOL participant sociodemographic and lifestyle characteristics were obtained by questionnaires conducted during the HCHS/SOL baseline visit. National background was determined by self-report and classified as: Mexican, Puerto Rican, Cuban, Dominican, Central American or South American. Alcohol use and tobacco use were determined by self-report and characterised as current, former or never. Education was described by three categories: less than high school, high school or equivalent or greater than high school. Household income was categorised into four groups: $<\$ 20000, \$ 20001-\$ 40000, \$ 40001-\$ 75000$ or $>\$ 75$ 000. USA-born was defined as born in the 50 United States, excluding US territories. Physical activity was determined using the Global Physical Activity Questionnaire and was classified as low, moderate or high.

\section{Statistical methods}

For this cross-sectional analysis, we included only participants without coronary artery disease or severe aortic or mitral valve disease who report no prior or current tobacco smoking. Coronary artery disease was determined by self-report or pathological Q-waves on ECG consistent with prior myocardial infarction. Aortic and mitral valve disease was determined by echocardiography. The baseline characteristics of participants with and without self-reported chronic household exposure to SHS were compared. The corresponding distribution of all baseline sociodemographic and clinical characteristics was summarised for the overall population using means \pm SEs for continuous variables and proportions for categorical variables. The mean values of the echocardiographic outcome variables in participants with and without SHS exposure were compared using t-tests.

Multivariable linear regression analysis was conducted comparing echocardiographic measures of cardiac structure and function in individuals who reported any childhood or adolescent/adult exposure to SHS to individuals who did not report any exposure to SHS. Linear regression models adjusted for the following potential confounding
Table 1 Population characteristics according to household secondhand tobacco smoke exposure

\begin{tabular}{|lcc|}
\hline & $\begin{array}{l}\text { Any household } \\
\text { secondhand } \\
\text { tobacco smoke } \\
\text { exposure } \\
\text { (N=615) }\end{array}$ & $\begin{array}{l}\text { No household } \\
\text { secondhand } \\
\text { tobacco smoke } \\
\text { exposure } \\
\text { (N=454) }\end{array}$ \\
\hline Age, mean (SE) & $55.5(0.5)$ & $56.9(1.0)$ \\
\hline Female sex, N (weighted \%) & $455(68.4)$ & $331(67.7)$ \\
\hline National background, N (weighted \%) & $151(25.4)$ & $78(17.5)$ \\
\hline Dominican & $92(12.2)$ & $71(22.6)$ \\
\hline Puerto Rican & $146(20.3)$ & $140(23.0)$ \\
\hline Mexican & $113(30.4)$ & $51(21.2)$ \\
\hline Cuban & $57(5.7)$ & $68(8.2)$ \\
\hline Central American & $56(6.0)$ & $45(7.5)$ \\
\hline South American & $327(52.0)$ & $232(53.5)$ \\
\hline Low physical activity, N (weighted \%) & $165(30.9)$ \\
\hline Education level, N (weighted \%) & $216(32.2)$ & $92(15.1)$ \\
\hline Less than high school & $132(21.7)$ & $196(53.9)$ \\
\hline High school or equivalent & $266(46.2)$ & $205(46.5)$ \\
\hline Greater than high school & $301(52.8)$ & $416(93.4)$ \\
\hline $\begin{array}{l}\text { Household income less than \$20 000 } \\
\text { per year, N (weighted \%) }\end{array}$ & $575(92.0)$ & \\
\hline Not US mainland born, N (weighted \%) & & \\
\hline
\end{tabular}

variables: model 1: age and sex; model 2: age, sex, study site, alcohol use, physical activity and years of education. Additionally, linear regression analyses were conducted to compare participants who have no reported chronic household SHS exposure to participants in each of the following categories of SHS exposure: childhood exposure only, adolescent/adult exposure only and both childhood and adolescent/adult exposure. Adjustment for potential confounding variables was conducted as specified above.

Current exposure to SHS was assessed in linear regression analyses comparing echocardiographic measures of cardiac structure and function in individuals who currently have no members of the household who smoke tobacco regularly with individuals who report 1 household smoker and/or 2+ household smokers. Additional linear regression analyses were conducted to assess the association between the number of hours per week spent in close contact with smokers in a closed space and echocardiographic measures of cardiac structure and function. Individuals who report 0 hours per week exposed with an active smoker in a closed space were compared with individuals who reported 1 hour to the median number of hours per week and to individuals who reported above the median number of hours per week. Adjustment for potential confounding variables was conducted as specified above. All analyses used sample weights to account for sampling probability and non-response and to make the estimates applicable to the target population based on guidelines suggested by the HCHS/ SOL Steering and Data Analysis Committees. Statistical analyses were completed using SAS V.9.3. 
Table 2 Association of any childhood or adolescent/adult household secondhand tobacco smoke exposure with echocardiographic measures of cardiac structure and function

\begin{tabular}{lllll} 
& $\begin{array}{l}\text { Minimally adjusted } \\
\boldsymbol{\beta} \text { (SE) }\end{array}$ & P values & $\begin{array}{l}\text { Fully adjusted } \\
\boldsymbol{\beta} \text { (SE) }\end{array}$ & P values \\
\hline LV mass index, gm/m & $1.7(1.9)$ & 0.38 & $1.4(1.8)$ & 0.42 \\
\hline LV end-systolic volume, $\mathrm{mL}$ & $-1.4(1.0)$ & 0.15 & $-1.2(0.8)$ & 0.14 \\
\hline LV end-diastolic volume, $\mathrm{mL}$ & $-3.6(2.0)$ & 0.07 & $-3.2(1.7)$ & 0.05 \\
\hline LVEF, \% & $0.3(0.4)$ & 0.57 & $0.3(0.4)$ & 0.51 \\
\hline LV stroke volume, $\mathrm{mL}$ & $0.2(1.4)$ & 0.86 & $0.5(1.3)$ & 0.71 \\
\hline LV longitudinal strain (four-chamber), \% & $0.1(0.3)$ & 0.84 & $0.02(0.3)$ & 0.94 \\
\hline LV longitudinal strain (two-chamber), \% & $0.9(0.5)$ & 0.07 & $0.8(0.4)$ & 0.06 \\
\hline LV longitudinal strain average), \% & $0.5(0.3)$ & 0.10 & $0.4(0.3)$ & 0.12 \\
\hline Medial E' velocity, cm/s & $-0.2(.1)$ & 0.19 & $-0.1(0.1)$ & 0.31 \\
\hline Lateral E' velocity, cm/s & $-0.2(0.3)$ & 0.50 & $-0.07(0.2)$ & 0.77 \\
\hline E/E' & $0.1(0.3)$ & 0.67 & $0.1(0.3)$ & 0.63 \\
\hline E/A ratio & $-0.02(0.02)$ & 0.43 & $-0.02(0.02)$ & 0.41 \\
\hline Isovolumic relaxation time, $\mathrm{s}$ & $0.001(0.002)$ & 0.45 & $0.001(0.001)$ & 0.34 \\
\hline Peak RA/RV gradient, mm Hg & $0.4(0.6)$ & 0.54 & $0.5(0.5)$ & 0.36 \\
\hline TAPSE, cm & $-0.04(0.05)$ & 0.45 & $-0.02(0.04)$ & 0.57 \\
\hline RV fractional area change, \% & $-0.01(0.01)$ & 0.25 & $-0.007(0.009)$ & 0.39 \\
\hline Left atrial volume index, $\mathrm{mL} / \mathrm{m}^{2}$ & $0.6(0.5)$ & 0.24 & $0.7(0.5)$ & 0.13 \\
\hline
\end{tabular}

Linear regression analyses adjusting for the following variables.

Minimally adjusted: age and sex.

Fully adjusted: age, sex, study site, alcohol use, physical activity and years of education.

E/A, E/A velocity; E/E', E/E'velocity ratio; LV, left ventricular; LVEF, left ventricular ejection fraction; RA, right atrial; RV, right ventricular;

TAPSE, tricuspid annular plane systolic excursion.

\section{RESULTS}

A total of 1069 never smokers $(68 \%$ women, mean age 56 years) were included in this analysis (table 1 ). Individuals who reported chronic household SHS exposure were disproportionately of Cuban and Dominican national background. Chronic household SHS exposure was also associated with decreased educational attainment and household income less than $\$ 20000$ per year. Individuals who reported any prior chronic childhood or adolescent/ adult household SHS exposure did not differ in any echocardiographic measure of cardiac structure and function when compared with individuals who did not report any chronic exposure to household SHS (table 2). However, in assessing childhood and adolescent/adult exposures individually, exposure to household SHS smoke in childhood only was associated with decreased $\mathrm{E} / \mathrm{A}$ ratio $(-0.06$ ( $\mathrm{SE}$ 0.02 ), $p=0.008$ ), after adjusting for covariates (table 3 ). In the fully adjusted model, exposure to household SHS in adolescent/adulthood only was associated with increased LVEF (1.2\% (0.6), $\mathrm{p}=0.04)$, increased left atrial volume index (1.8 (0.9), $\mathrm{p}=0.045)$ and decreased IVRT $(-0.003 \mathrm{~s}$ (0.002), $\mathrm{p}=0.03)$. Individuals who reported household SHS exposure in both childhood and adolescence/adulthood had worse LV longitudinal strain (two-chamber) compared with individuals who reported no household SHS exposure $(0.8 \%(0.4), p=0.049)$ after adjustment for covariates.
Current household secondhand tobacco smoke exposure as determined by the number of smokers who regularly smoke tobacco in the home was associated with worse left and right heart function (table 4). Individuals who report one household smoker had reduced LVEF $(-1.4 \%$ (0.6), $\mathrm{p}=0.02$ ), worse LV longitudinal strain (four-chamber and average) $(1.1 \%(0.4), \mathrm{p}=0.01 \%$ and $0.9 \%(0.4), \mathrm{p}=0.03$, respectively), reduced medial $\mathrm{E}^{\prime}$ velocity $(-0.5 \mathrm{~m} / \mathrm{s}(0.20)$, $\mathrm{p}=0.01)$, reduced $\mathrm{E} / \mathrm{A}$ ratio $(-0.09(0.03), \mathrm{p}=0.003)$ and reduced right ventricular fractional area change $(-0.02 \%$ $(0.01), p=0.01)$. IVRT was increased in individuals who reported one household smoker $(0.006 \mathrm{~s}(0.003), \mathrm{p}=0.04)$. Individuals who reported two or more household smokers $(\mathrm{N}=31)$ had reduced $\mathrm{E} / \mathrm{E}^{\prime}$ ratio $(-1.6(0.6), \mathrm{p}=0.0155)$. No association between the reported number of hours per week exposed to secondhand tobacco smoke and cardiac structure and function was observed (online supplementary table 1).

\section{DISCUSSION}

In a representative sample of Hispanics/Latinos, chronic exposure to SHS was associated with changes in cardiac structure along with systolic and diastolic functions. Chronic SHS exposure in childhood only was associated with worse LV diastolic function, while chronic SHS 


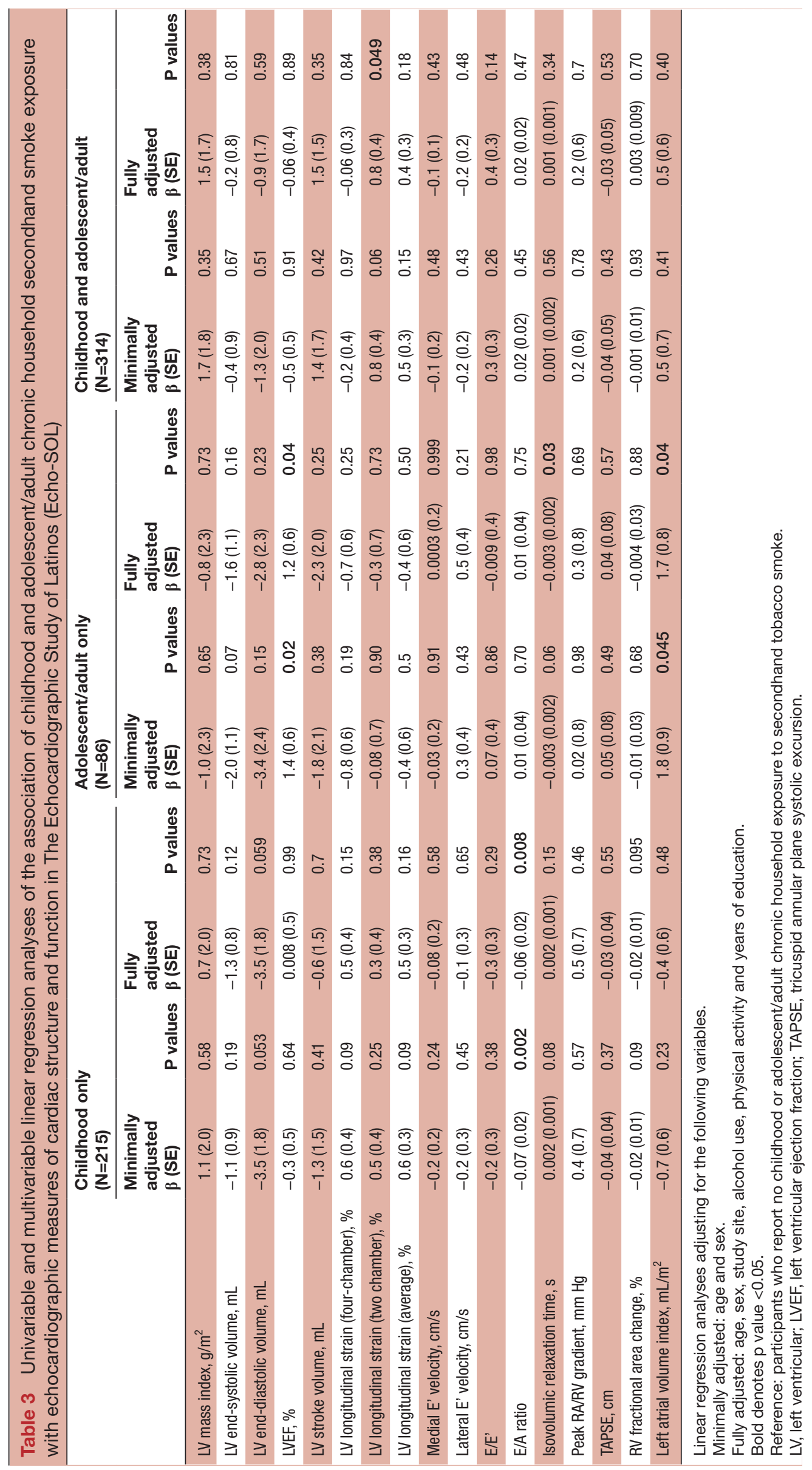


Table 4 Association of current number of household smokers that regularly smoke in the home and echocardiographic measures of cardiac structure and function in Echo-SOL

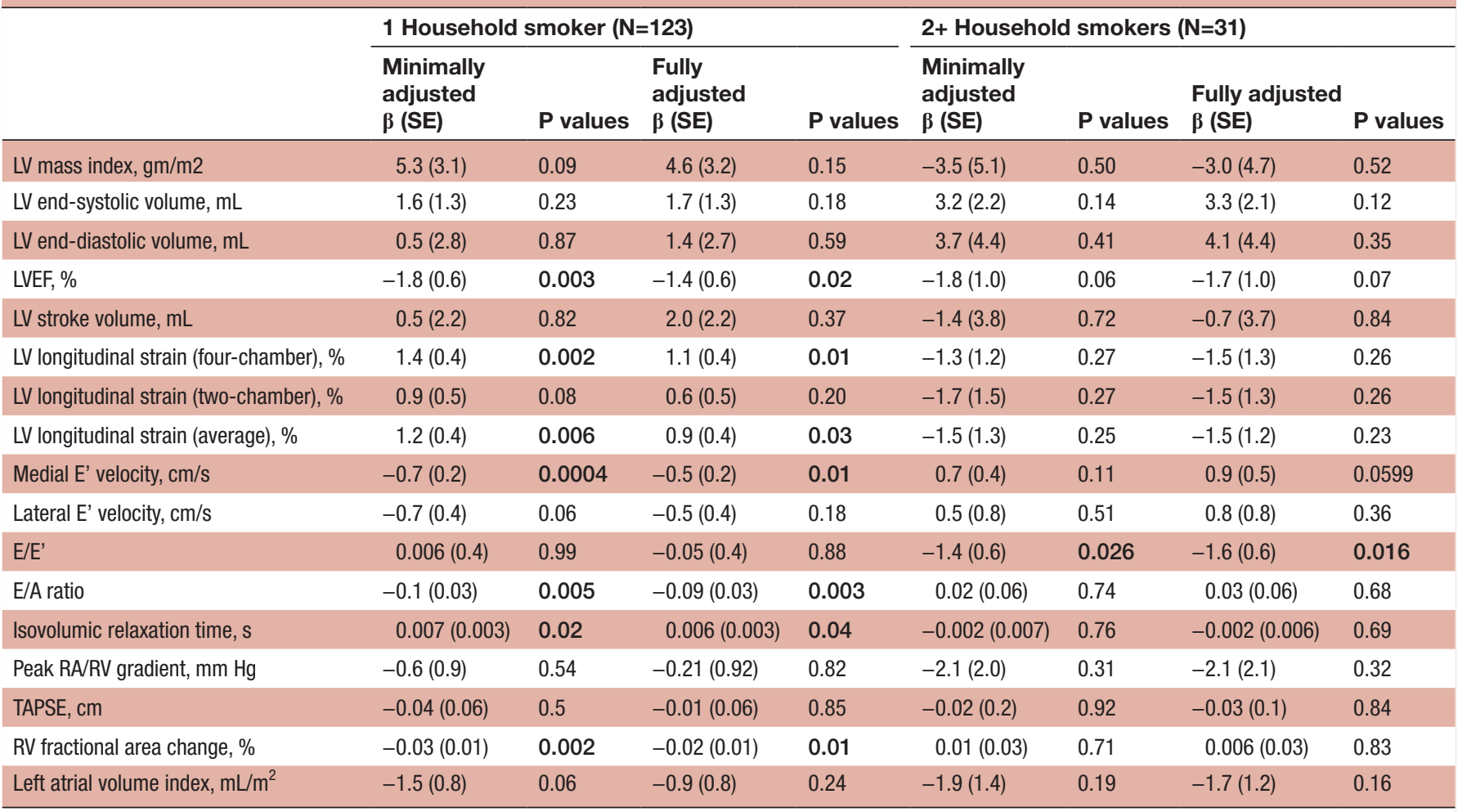

Linear regression analyses adjusting for the following variables.

Minimally adjusted: age and sex.

Fully adjusted: age, sex, study site, alcohol use, physical activity and years of education.

Bold denotes $p$ value $<0.05$.

Reference: participants who currently do not live with a tobacco smoker who regularly smokes in the home.

LV, left ventricular; LVEF, left ventricular ejection fraction; TAPSE, tricuspid annular plane systolic excursion.

exposure in adolescence/adulthood only was associated with increased left atrial size and shorter IVRT signalling reduced LV compliance causing a rapid increase in LV intracardiac pressures with early cessation of LV filling. Chronic SHS exposure in adolescence/adulthood was also paradoxically increased LVEF possibly as a means of compensation to maintain cardiac output in the setting of abnormal diastolic function. Chronic SHS exposure in both childhood and adolescence/adulthood was associated with decreased myocardial deformation consistent with a pattern seen in early heart failure with preserved ejection fraction (HFpEF) where there is abnormal diastolic function, normal systolic function but abnormal myocardial deformation mechanics. ${ }^{22}$ Current SHS exposure from living with a tobacco smoker was associated with decreased LV systolic function as measured by ejection fraction and global longitudinal strain.

To our knowledge, this study represents one of the first studies to examine the relationship of chronic SHS exposure with cardiac structure and function. The results of this study contribute to the emerging body of evidence regarding the adverse effects of exposure to tobacco smoke on cardiac structure and function, which has primarily been studied in active current smokers. Within Echo-SOL, increased packyears of tobacco smoking was associated with decreased systolic and diastolic function and increased cigarettes smoked per day were associated with increased LV mass. ${ }^{23}$ A study of the Coronary Artery Risk Development in Young Adults (CARDIA) cohort found that tobacco smoking was associated with a trend towards increased LV mass in all groups except for African-American men and increased LV stress in women; however, the echocardiographic variables in this study were limited to linear measurements, and 2D quantitation was not performed. ${ }^{24}$ In Multi-Ethnic Study of Atherosclerosis (MESA), a dose-response relationship between cigarette consumption measured in pack-years and regional LV dysfunction was noted. ${ }^{25}$ Furthermore, a controlled experiment of the acute response to active tobacco smoking demonstrated immediate impairment in multiple measures of diastolic function. ${ }^{26}$ However, the data from our study support that notion that even passive SHS is associated with alterations LV systolic function and diastolic function.

The mechanisms by which tobacco smoke exposure impacts myocardial function are not well described, although exposure to SHS has been associated with increased serum homocysteine and fibrinogen levels, which are associated with increased inflammation and thrombosis. ${ }^{27}$ Experimental and clinical studies have shown the direct toxic effects of cigarette smoke on the myocardium, including abnormalities in energy metabolism, lipotoxicity and oxidative stress. ${ }^{28}{ }^{29}$ Additionally, animal models 
suggest that chronic exposure to tobacco smoke increases blood pressure and oxidative stress and causes endothelial dysfunction. ${ }^{30}$ Of note, in our study, current exposure to a household smoker was associated with more profound decreases in systolic and diastolic function than any other measure of chronic SHS smoke exposure. Yet, in the absence of current exposure to SHS, chronic SHS exposure in childhood was still associated with decrease diastolic function, thus highlighting the potential long-term impact of remote SHS exposure on cardiac function. In utero exposure to maternal and paternal smoking has been found to increase the risk of decreased cardiac function in children. ${ }^{31-34} \mathrm{In}$ our study, it is difficult to determine whether participants that report childhood exposure to SHS were also exposed in utero and thus separate the cardiac effects of in utero exposure from childhood SHS exposure. However, these findings are hypothesis generating and highlight the potential impact of childhood environmental exposures on future cardiac health in adulthood.

The observed relationship between SHS exposure and cardiac function has implications for public health strategies for primordial prevention of heart failure, particularly in Hispanics/Latinos. During 2011-2012, about 58 million non-smokers in the USA were exposed to SHS; with two out of every five children-including 7 out of every 10 black children-being exposed to SHS regularly. ${ }^{17}$ Racial and ethnic differences in LV mass, diastolic function, systolic function and myocardial deformation are not completely explained by cardiometabolic risk factors and comorbidities. ${ }^{35}$ Within Echo-SOL, the average values of LV size and mass are different than what has been reported in non-Hispanic white cohorts of healthy participants. ${ }^{36}$ Additionally, LV diastolic dysfunction is more highly prevalent in Hispanics/Latinos than in cohorts of non-Hispanic whites. ${ }^{37}$ Similar differences have been observed in reference values for global longitudinal strain in healthy African-Americans when compared with healthy whites in the CARDIA study. ${ }^{38}$ The degree to which intervenable environmental exposures, such as household exposure to SHS, might contribute to racial/ethnic differences in cardiac function, and thus future heart failure risk, is an important consideration. $^{39}$

This study has several limitations. First, SHS exposure was assessed by self-report and thus is subject to recall bias. Second, considerable heterogeneity in SHS exposure intensity for each measure of SHS likely exists. Serum cotinine levels were not measured to quantitatively assess current SHS exposure and assess for dose response. Third, the cross-sectional design of this observational study limits our ability to determine causality. We acknowledge that we did not account for multiple testing. However, most of our findings are at the $\mathrm{p}$ value less than the 0.01 level. SHS is conceptualised as a form of indoor air pollution, ${ }^{40}$ placing hypertension, obesity and diabetes ${ }^{41}$ on the causal pathway of our cardiac outcomes and thus justifying their exlusion fromour statistical models as confounders. Finally, the sample size of Echo-SOL is modest, limiting statistical power and type II error. Future studies of SHS and cardiac function with a larger sample and a longitudinal design are needed to better assess cardiac function and clinical heart failure outcomes.

\section{CONCLUSION}

In Hispanics/Latinos, chronic SHS smoke exposure in childhood was associated with decreased LV diastolic function. Living with a tobacco smoker who smokes in the home was associated with abnormal diastolic function, abnormal systolic function and abnormal myocardial deformation. While it is known that subclinical LV dysfunction increases risk for clinical heart failure, ${ }^{42}$ the relationship between chronic SHS exposure and incident heart failure warrants further investigation in future studies. Reducing childhood and adulthood exposure to SHS in the home is a potential opportunity for primordial prevention of heart failure, particularly among Hispanics/Latinos.

\section{Author affiliations}

${ }^{1}$ Stanford Health Care, Oakland, California, USA

2Department of Medicine, Division of Cardiology, Wake Forest School of Medicine, Winston-Salem, North Carolina, USA

${ }^{3}$ Department of Epidemiology and Population Health, Albert Einstein College of Medicine, Bronx, New York, USA

${ }^{4}$ Deparment of Social Medicine, University of North Carolina at Chapel Hill School of Medicine, Chapel Hill, North Carolina, USA

${ }^{5}$ Institute for Minority Health Research, Unverisity of Illinois School of Medicine, Chicago, Illinois, USA

${ }^{6}$ Department of Medicine, School of Medicine, University of Illinois, Chicago, Illinois, USA

${ }^{7}$ Department of Biostatistics, Gillings School of Global Public Health, University of North Carolina at Chapel Hill, Chapel Hill, North Carolina, USA

${ }^{8}$ Department of Preventive Medicine, Feinberg School of Medicine, Northwestern Univerisity, Chicago, Illinois, USA

${ }^{9}$ Department of Psychology, Miller School of Medicine, University of Miami, Miami, Florida, USA

${ }^{10}$ Department of Medicine, Yale School of Medicine, New Haven, Connecticut, USA

Acknowledgements The authors would like to acknowledge the investigators, the staff and the participants of HCHS-SOL and Echo-SOL for their dedication and commitment to the success of this study. Investigators' website: http://www.cscc. unc.edu/hchs/.

Contributors All authors fulfil the International Committee of Medical Journal Editors recommendations for authorship. Conceived and designed the research: MSBP and CJR. Performed statistical analysis: KS, MSBP and CJR. Interpretation of statistical analysis: KS, MSBP and CJR. Data acquisition: RCK, KP, MD, JC, ALG MDG abd CJR. Drafted the manuscript: MSBP and CJR. Made critical revision of the manuscript for key intellectual content: MSBP, RCK, KP, MD, JC, MMK, EJV, ALG, $M D G$ and CJR.

Funding The Hispanic Community Health Study/Study of Latinos (HCHS/SOL) was performed as a collaborative study supported by contracts from the National Heart, Lung, and Blood Institute (NHLBI) to the University of North Carolina (N01- HC65233), University of Miami (N01-HC65234), Albert Einstein College of Medicine (N01HC65235), Northwestern University (N01-HC65236) and San Diego State University (N01-HC65237). The following institutes/centres/offices contribute to the HCHS/ SOL through a transfer of funds to the NHLBI: National Institute on Minority Health and Health Disparities, National Institute on Deafness and Other Communication Disorders, National Institute of Dental and Craniofacial Research, National Institute of Diabetes and Digestive and Kidney Diseases, National Institute of Neurological Disorders and Stroke and National Institutes of Health Institution-Office of Dietary Supplements. Echo-S0L was supported by a grant from the NHLBI (R01 HL104199, Epidemiological Determinants of Cardiac Structure and Function among Hispanics: CJR as principal investigator).

Competing interests None declared. 
Patient consent Obtained.

Ethics approval Wake Forest School of Medicine.

Provenance and peer review Not commissioned; externally peer reviewed.

Open access This is an open access article distributed in accordance with the Creative Commons Attribution Non Commercial (CC BY-NC 4.0) license, which permits others to distribute, remix, adapt, build upon this work non-commercially, and license their derivative works on different terms, provided the original work is properly cited, appropriate credit is given, any changes made indicated, and the use is non-commercial. See: http://creativecommons.org/licenses/by-nc/4.0/

\section{REFERENCES}

1. Oberg M, Jaakkola MS, Woodward A, et al. Worldwide burden of disease from exposure to second-hand smoke: a retrospective analysis of data from 192 countries. Lancet 2011;377:139-46.

2. Giraldi G, Fovi De Ruggiero G, Marsella LT, et al. Environmental tobacco smoke: health policy and focus on Italian legislation. Clin Ter 2013;164:e429-35.

3. Sebrié EM, Sandoya E, Hyland A, et al. Hospital admissions for acute myocardial infarction before and after implementation of a comprehensive smoke-free policy in Uruguay. Tob Control 2013;22(e1):e16-e20.

4. Barnoya J, Glantz SA. Cardiovascular effects of secondhand smoke: nearly as large as smoking. Circulation 2005;111:2684-98.

5. Gallo V, Neasham D, Airoldi L, et al. Second-hand smoke, cotinine levels, and risk of circulatory mortality in a large cohort study of never-smokers. Epidemiology 2010;21:207-14.

6. Lv X, Sun J, Bi Y, et al. Risk of all-cause mortality and cardiovascular disease associated with secondhand smoke exposure: a systematic review and meta-analysis. Int J Cardiol 2015;199:106-15.

7. Pope CA, Burnett RT, Krewski D, et al. Cardiovascular mortality and exposure to airborne fine particulate matter and cigarette smoke: shape of the exposure-response relationship. Circulation 2009;120:941-8.

8. Barnoya J, Glantz SA. Cardiovascular effects of second-hand smoke help explain the benefits of smoke-free legislation on heart disease burden. J Cardiovasc Nurs 2006;21:457-62.

9. Hurt RD, Weston SA, Ebbert JO, et al. Myocardial infarction and sudden cardiac death in Olmsted County, Minnesota, before and after smoke-free workplace laws. Arch Intern Med 2012;172:1635-41.

10. Johnson EL, Beal JR. Impact of a comprehensive smoke-free law following a partial smoke-free law on incidence of heart attacks at a rural community hospital. Nicotine Tob Res 2013;15:745-7.

11. Lippert WC, Gustat J. Clean indoor air acts reduce the burden of adverse cardiovascular outcomes. Public Health 2012;126:279-85.

12. Naiman A, Glazier RH, Moineddin R. Association of anti-smoking legislation with rates of hospital admission for cardiovascular and respiratory conditions. CMAJ 2010;182:761-7.

13. Daviglus ML, Talavera GA, Avilés-Santa ML, et al. Prevalence of major cardiovascular risk factors and cardiovascular diseases among Hispanic/Latino individuals of diverse backgrounds in the United States. JAMA 2012;308:1775-84.

14. Navas-Nacher EL, Kelley MA, Birnbaum-Weitzman O, et al. Association between exposure to household cigarette smoking behavior and cigarette smoking in Hispanic adults: findings from the hispanic community health study/study of Latinos. Prev Med 2015;77:35-40.

15. Pérez-Stable EJ, Ramirez A, Villareal R, et al. Cigarette smoking behavior among US Latino men and women from different countries of origin. Am J Public Health 2001;91:1424-30.

16. Joya X, Manzano C, Álvarez AT, et al. Transgenerational exposure to environmental tobacco smoke. Int J Environ Res Public Health 2014;11:7261-74.

17. Homa DM, Neff LJ, King BA, et al. Vital signs: disparities in nonsmokers' exposure to secondhand smoke--United States, 19992012. MMWR Morb Mortal Wkly Rep 2015;64:103-8.

18. Vivo RP, Krim SR, Krim NR, et al. Care and outcomes of Hispanic patients admitted with heart failure with preserved or reduced ejection fraction: findings from get with the guidelines-heart failure. Circ Heart Fail 2012;5:167-75.

19. Lavange LM, Kalsbeek WD, Sorlie PD, et al. Sample design and cohort selection in the Hispanic Community Health Study/Study of Latinos. Ann Epidemiol 2010;20:642-9.

20. Sorlie PD, Avilés-Santa LM, Wassertheil-Smoller S, et al. Design and implementation of the Hispanic Community Health Study/Study of Latinos. Ann Epidemiol 2010;20:629-41.
21. Rodriguez CJ, Dharod A, Allison MA, et al. Rationale and design of the Echocardiographic Study of Hispanics/Latinos (ECHO-SOL). Ethn Dis 2015;25:180-6.

22. Kraigher-Krainer E, Shah AM, Gupta DK, et al. Impaired systolic function by strain imaging in heart failure with preserved ejection fraction. J Am Coll Cardiol 2014;63:447-56.

23. Leigh JA, Kaplan RC, Swett K, et al. Smoking intensity and duration is associated with cardiac structure and function: the ECHOcardiographic Study of Hispanics/Latinos. Open Heart 2017;4:e000614.

24. Gidding SS, Xie X, Liu K, et al. Cardiac function in smokers and nonsmokers: the CARDIA study. The Coronary Artery Risk Development in Young Adults Study. J Am Coll Cardiol 1995;26:211-6.

25. Rosen BD, Saad MF, Shea S, et al. Hypertension and smoking are associated with reduced regional left ventricular function in asymptomatic: individuals the Multi-Ethnic Study of Atherosclerosis. J Am Coll Cardiol 2006;47:1150-8.

26. Farsalinos KE, Tsiapras D, Kyrzopoulos S, et al. Acute effects of using an electronic nicotine-delivery device (electronic cigarette) on myocardial function: comparison with the effects of regular cigarettes. BMC Cardiovasc Disord 2014;14:78.

27. Venn A, Britton J. Exposure to secondhand smoke and biomarkers of cardiovascular disease risk in never-smoking adults. Circulation 2007:115:990-5.

28. Santos PP, Oliveira F, Ferreira VC, et al. The role of lipotoxicity in smoke cardiomyopathy. PLoS One 2014;9:e113739.

29. Kaplan A, Abidi E, Ghali R, et al. Functional, cellular, and molecular remodeling of the heart under influence of oxidative cigarette tobacco smoke. Oxid Med Cell Longev 2017;2017:1-16.

30. Talukder MA, Johnson WM, Varadharaj S, et al. Chronic cigarette smoking causes hypertension, increased oxidative stress, impaired NO bioavailability, endothelial dysfunction, and cardiac remodeling in mice. Am J Physiol Heart Circ Physiol 2011;300:H388-H396.

31. Treyster Z, Gitterman B. Second hand smoke exposure in children: environmental factors, physiological effects, and interventions within pediatrics. Rev Environ Health 2011;26:187-95.

32. Bakker H, Jaddoe VW. Cardiovascular and metabolic influences of fetal smoke exposure. Eur J Epidemiol 2011;26:763-70.

33. Banderali G, Martelli A, Landi M, et al. Short and long term health effects of parental tobacco smoking during pregnancy and lactation: a descriptive review. J Trans/ Med 2015;13:327.

34. Taal HR, de Jonge LL, van Osch-Gevers L, et al. Parental smoking during pregnancy and cardiovascular structures and function in childhood: the Generation R Study. Int J Epidemiol 2013;42:1371-80.

35. Rodriguez CJ, Diez-Roux AV, Moran A, et al. Left ventricular mass and ventricular remodeling among Hispanic subgroups compared with non-Hispanic blacks and whites: MESA (Multi-ethnic Study of Atherosclerosis). J Am Coll Cardiol 2010;55:234-42.

36. Qureshi WT, Leigh JA, Swett K, et al. Comparison of echocardiographic measures in a Hispanic/Latino Population With the 2005 and 2015 American Society of Echocardiography Reference Limits (The Echocardiographic Study of Latinos). Circ Cardiovasc Imaging 2016;9.

37. Mehta H, Armstrong A, Swett K. Burden of systolic and diastolic left ventricular dysfunction among hispanics of Systolic and Diastolic Left Ventricular Dysfunction Among Hispanics in the United States: insights from the echocardiographic study of Latinos. Circ Heart Fail 2016;9:e002733.

38. Moreira HT, Nwabuo CC, Armstrong AC. Reference ranges and regional patterns of left ventricular strain and strain rate using two-dimensional speckle-tracking echocardiography in a healthy middle-aged black and white population: the cardia stence Ranges and regional patterns of left ventricular strain and strain rate using two-dimensional speckle-tracking echocardiography in a healthy middle-aged black and white population: the CARDIA Study. J Am Soc Echocardiogr 2017;30:647-58.

39. Cho GY, Marwick TH, Kim HS, et al. Global 2-dimensional strain as a new prognosticator in patients with heart failure. J Am Coll Cardiol 2009;54:618-24.

40. Butz AM, Breysse P, Rand C, et al. Household smoking behavior: effects on indoor air quality and health of urban children with asthma. Matern Child Health J 2011;15:460-8.

41. Wei Y, Zhang JJ, Li Z, et al. Chronic exposure to air pollution particles increases the risk of obesity and metabolic syndrome: findings from a natural experiment in Beijing. Faseb $J$ 2016;30:2115-22.

42. Echouffo-Tcheugui JB, Erqou S, Butler J. Assessing the risk of pro Progression from asymptomatic left ventricular dysfunction to overt heart failure: a systematic overview and meta-analysis. JACC Heart Fail 2016;4:237-48. 